\title{
Experimental and Numerical Studies on the Dynamic Behaviors of Concrete Material Based on the Waveform Features in SHPB Test
}

\author{
Xiao Weintu Chen ${ }^{1 *}$, Taihong Lv ${ }^{2,3}$, Gang Chen $^{3}$ \\ ${ }^{1}$ State Key Laboratory of Explosion Science and Technology, Beijing Institute of Technology, Beijing 100081, China \\ ${ }^{2}$ Department of Modern Mechanics, University of Science and Technology of China, Hefei, Anhui 230027, China \\ ${ }^{3}$ Institute of Systems Engineering, China Academy of Engineering Physics, Mianyang, Sichuan 621999, China
}

\begin{abstract}
The tendency of the waveform curve can directly reflect the deformation and failure process of specimen in the SHPB (Split Hopkinson Pressure Bar) test of concrete. Different loading rates will result in the different ultimate failure modes, waveform curves. Furthermore, these differences are obviously characterized by some feature points of waveform or stress-strain curves. It is to say for concrete-like damage softening materials, the waveform features contains lots of information of material response. In this study, large dimension $(\Phi 120 \mathrm{~mm})$ SHPB tests of concrete specimens have been conducted. Four typical failure patterns of concrete specimens are classified, as well as some typical waveform features, e.g. the "double-peak" and "compression wave" phenomena of reflection wave, etc. On the other hand, the numerical simulations corresponding to the experimental tests are performed by means of the $3 \mathrm{D}$ meso-scale model of concrete material. In the numerical results, waveform features observed in experiment are reliably reproduced and predicted. Associating with waveform features, the violation indicator of the specimen stress equilibrium in the SHPB test is first identified for concrete-like damage softening materials. The concrete material behaviors for stress non-equilibrium are further analyzed, e.g. DIF and damage development, etc.
\end{abstract}

\section{Introduction}

Concrete is a kind of material widely used in the civil and protective engineering, i.e. bridges, high-rise buildings, protective structures, etc. It is always required that these structures should have the capability of resisting the highrate loads, i.e. earthquake, impact, explosion, etc. Concrete is also a composite material with complicated internal components (consisting of coarse aggregates, mortar matrix and interface transition zone (ITZ)) and mechanical behaviors. Therefore, it is of important practical significance to investigate the dynamic behaviors of concrete material under the high-rate loading conditions [1-2].

Split Hopkinson pressure bar has been always used to study the dynamic performance of concrete by many researchers [3-6]. These researches mainly focus on the rate sensitivity of concrete in terms of a certain parameter, such as strength, elastic modulus, critical strain at the maximum stress or fracture energy, etc. However, in the previous studies, the analysis on the waveform features in SHPB tests of concrete is relatively less compared to that on the rate sensitivity. The waveform curves derived from different strain rates were always translated into the more intuitive stress-strain curves to study [3, 4]. Only in the analysis of the stress equilibrium for verifying the effectiveness of SHPB test results, very simple analysis of waveform curves might be reported. In other words, the feature analysis on the waveform curves under different strain rates, which directly reflects the deformation and failure process of concrete specimen, is more often to be neglected by researchers, as well as the material behaviors related to the waveform features.

\section{Fundamental principle of SHPB test}

The SHPB technique has been developed based on the theory of one-dimensional stress wave propagation in an elastic circular bar. Basically, the reliability of the experiment results of SHPB is determined by the following two assumptions: (1) one-dimensional stress wave assumption in the pressure bars; (2) the stresses and strains uniformly distributed along the length direction of specimen. Three waveforms are involved in the SHPB test, respectively are the incident compressive pulse, reflective tensile pulse and the transmission pulse. According to the one-dimensional stress wave theory, the stress, strain rate and strain can be expressed as follows, respectively:

$$
\sigma(\mathrm{t})=E\left(\frac{A}{A_{s}}\right) \varepsilon_{t}(t)
$$

\footnotetext{
*Corresponding author: chenxiaoweintu@yahoo.com
} 


$$
\begin{gathered}
\dot{\varepsilon}(t)=-\frac{2 C_{0}}{L} \varepsilon_{r}(t) \\
\varepsilon(t)=-\frac{2 C_{0}}{L} \int_{0}^{t} \varepsilon_{r}(\tau) d \tau
\end{gathered}
$$

Where $\varepsilon_{t}$ is the strain due to the transmitted pulse in the output bar, and $\varepsilon_{\mathrm{r}}$ is the strain due to the reflected pulse in the output bar. E, $\mathrm{A}$, and $\mathrm{C}_{0}$ are Young's modulus, the cross-sectional area and the elastic wave velocity of pressure bars, respectively. $\mathrm{A}_{\mathrm{s}}$ and $\mathrm{L}$ are the crosssectional area and length of specimen, respectively. As shown in Eq. (1), (2) and (3), in the signals measured in the SHPB test, it should be pointed out that transmission curve, reflection curve and the area below reflection curve are accorded with the relationships between stress, strain rates and strain versus time, respectively.

\section{Experimental results and feature analysis of waveform curves}

\subsection{Four typical failure patterns of concrete specimen}

Apparently, the ultimate failure patterns of concrete specimens are quite different under various impact velocities. According to the extent of damage and destruction, the ultimate failure patterns of concrete specimens can be simply classified into 4 scenarios, i.e., slight-spalling, breaking, fragmentation and comminution, respectively. For better understanding the waveform characteristics, four trials which can typically represent the failure patterns of concrete specimens in SHPB tests had been selected to analyze, as shown in Figure 1 (a-d). The corresponding velocities of the striker are $12.55 \mathrm{~m} / \mathrm{s}, 15.33 \mathrm{~m} / \mathrm{s}, 18.59 \mathrm{~m} / \mathrm{s}$ and $21.12 \mathrm{~m} / \mathrm{s}$, and the average strain rates are $13.27 / \mathrm{s}, 27.04 / \mathrm{s}, 55.4 / \mathrm{s}$ and $89.02 / \mathrm{s}$, respectively. Where, the average strain rate (ASR) is equal to the total strain accumulated in the loading process divided by the corresponding time.

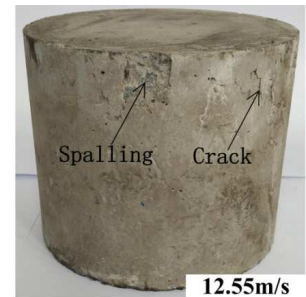

(a) Slight-spalling

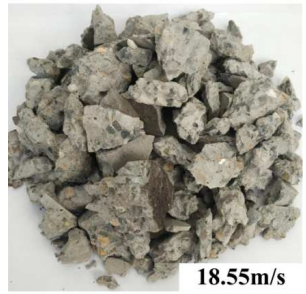

(c) Fragmentation

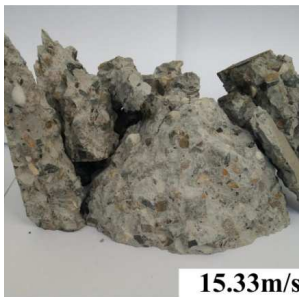

(b) Breaking

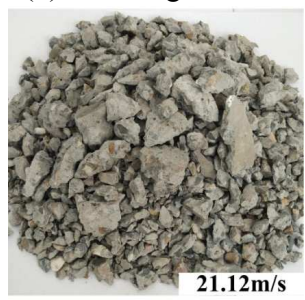

(d) Comminution
Fig. 1. Four Typical Failure Patterns of Concrete Specimen.

\subsection{The validation of stress equilibrium}

As we know, it is an important guarantee to achieve the longitudinal stress equilibrium in SHPB tests for the validity of test data $[7,8]$. According to the different ultimate failure patterns of concrete specimens, the above four groups of test data are checked, as shown in Figure 2 (a-d). It can be found that each test data shows better stress equilibrium. This indicates the experiment results of SHPB tests presented in this paper are valid.

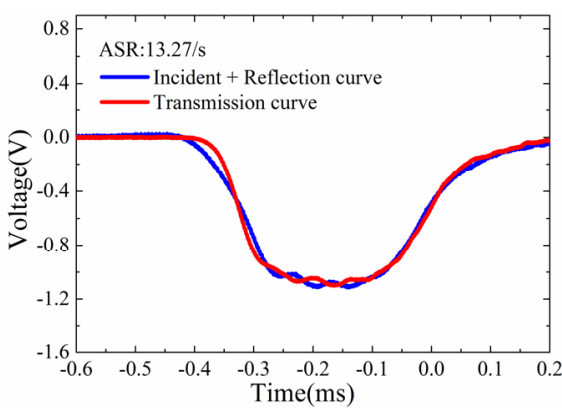

(a) Impact velocity is $12.55 \mathrm{~m} / \mathrm{s}$

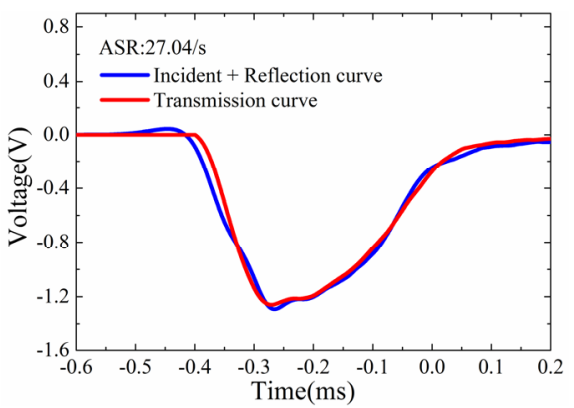

(b) Impact velocity is $15.33 \mathrm{~m} / \mathrm{s}$

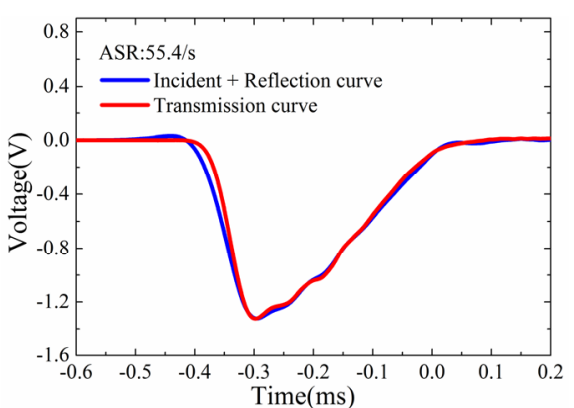

(c) Impact velocity is $18.58 \mathrm{~m} / \mathrm{s}$

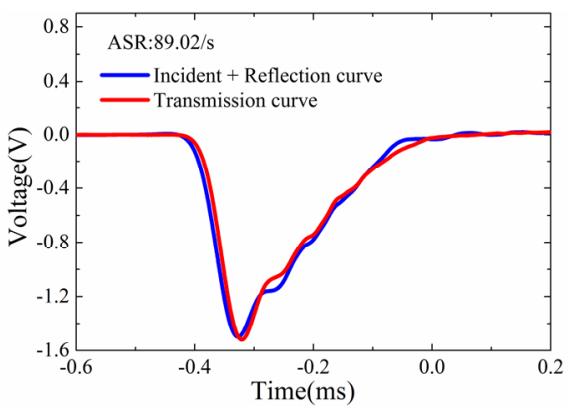

(d) Impact velocity is $21.12 \mathrm{~m} / \mathrm{s}$

Fig. 2. The validation of the stress equilibrium.

\subsection{Analysis on the "double-peak" phenomenon of reflection wave}

The experimental waveform curves corresponding to 
above four failure patterns are shown in Figure 3. In this figure, $\mathrm{O}$ is initial point and $\mathrm{C} \sim \mathrm{K}$ are seven feature points, one of which represents a particular time of the stress wave through the specimen.

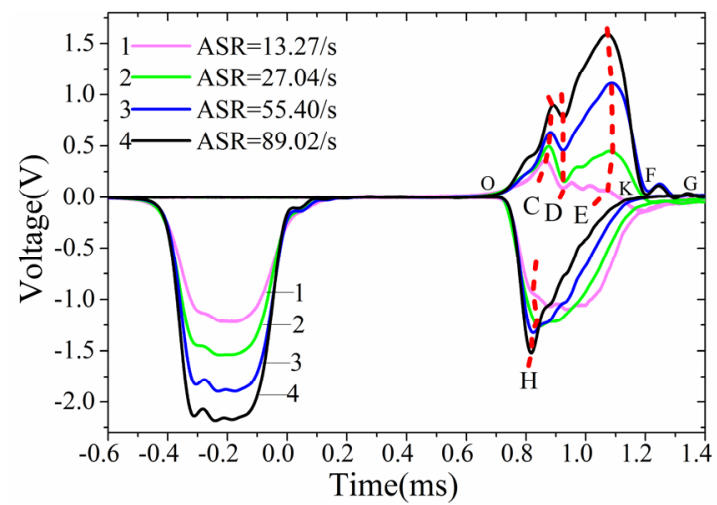

Fig. 3. Waveform curves under different strain rates.

With comparing the reflection waveform curves under different strain rates, it can be found that each reflection curve has a distinct "double-peak" phenomenon except the one in lowest strain rate (specimen slightly spalled, and considered to be almost intact) only has one peak. With the increase of impact velocity, the second peak is significantly higher than the first peak, and the difference between the first peak and the valley between the two peaks will gradually decrease.

Basically the "double-peak" phenomenon of the reflection wave comes from the damage of concrete specimen. When subjected to dynamic loading, concrete specimen suffers serious damage and material properties change significantly, and thus the specimen impedance will not match with that of the pressure bar seriously. It leads to only a little part of stress pulse transmitted to the output bar through the specimen. Instead, more part of the pulse can only be reflected back to the incident bar, so that the second peak is generated and the "double-peak" of reflection wave is developed. It can be deduced that the more serious the damage of concrete specimen is, the more incident stress pulse will be reflected to the input bar and the significantly higher second peak than the first peak will be resulted in, until the amplitude of the second peak reaches close to that of incident wave, and even forms a platform. Based on the above analysis, it can be assumed that the "double-peak" phenomenon of reflection wave is the specific property possessed by concrete-like brittle materials in SHPB tests.

\subsection{Analysis on the "compression wave" phenomenon in the tail of reflection wave}

Through comparing the reflection waveform curves under different strain rates, it is also can be found that the obvious compression wave occurs in FG section of reflection wave when the ultimate failure patterns show as "slight-spalling" or "breaking" (waveform 1 or 2, as shown in Figure 3).

In general, the reflection wave in the incident bar should fully show as the tension wave, and thus it is conflictive with that the "compression wave" phenomenon in the tail of reflection wave, which occurs between time points $\mathrm{F}$ and $\mathrm{G}$. Under the relatively lower impact velocity, the nearly intact or the larger residual cone of concrete specimen, as shown in Figure 3 (a-b), may suffer to certain unloading expansion recovery and then the input bar will be compressed. Therein the compression section FG in reflection wave will be generated and the corresponding stress-strain curve will behave as approximately linear elastic unloading. With the increase of the impact velocity, the concrete specimen will be seriously destructed and the size of the residual end cone will be decreased and even the residual cone vanishes. The compression portion of reflection wave is considered to be the necessary phenomenon in the SHPB test of metal specimen. Regarding the concrete-like brittle material specimen in SHPB tests, "compression wave" phenomenon in the tail of reflection wave exists only in the case that there left large residual specimen after SHPB test.

\section{Numerical results and discussion for material dynamic behaviors of concrete}

\subsection{D meso-scale model of concrete specimen}

In the numerical investigation of this paper, the aggregates, mortar and ITZ are considered to create the meso-scale model of concrete specimen, as shown in Figure $4(a-b)$. Figure $4(a)$ contains a section view of the model specimen and the zoom of the local mesh grid. Figure 4 (b) exhibits the distribution of the four-gradation aggregates, the volume fractions of the aggregates is about $41.2 \%$, and the particle size ranges with respect to each gradation are 20 16、16 12、12 8 and $8 \sim 4 \mathrm{~mm}$.

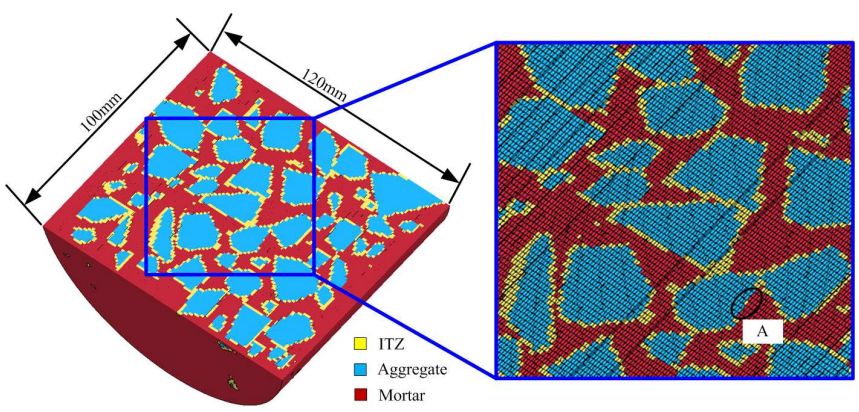

(a) The section view of the model specimen and the zoom of

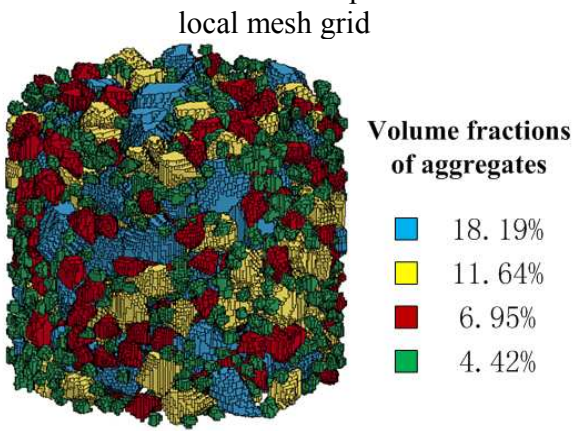

(b) The distribution of the four-gradation aggregates

Fig. 4. The 3D meso-scale finite element model of concrete specimen. 


\subsection{Material parameters of HJC model}

The LS-DYNA material model of JOHNSON HOLMQUIST CONCRETE (HJC, Mat 111) 9 proposed by Holmquist, Johnson and Cook in 1993 is adopted to model the material behaviors of the mortar, aggregates and ITZ in the numerical study of this paper. A set of HJC material parameters of mortar, ITZ and aggregates is determined by Lv et al. [10], and it makes the predicted results well agree with the experimental observations. The material parameters by Lv et al. [10] are adopted in this study.

\subsection{The validation of the numerical model}

In most numerical investigations of SHPB test of concrete specimen, e.g. [11-12], the primary validation of the numerical model is always achieved by rendering the numerical waveforms to be nearly consistent with the experimental ones. When the striker velocity is 18.58 $\mathrm{m} / \mathrm{s}$, the numerical and experimental waveform curves are given in Figure 5. Obviously, the numerical SHPB test shows good agreement with the experimental data. Therefore, the simulations based on the meso-scale concrete model generated in this paper are with good reliability.

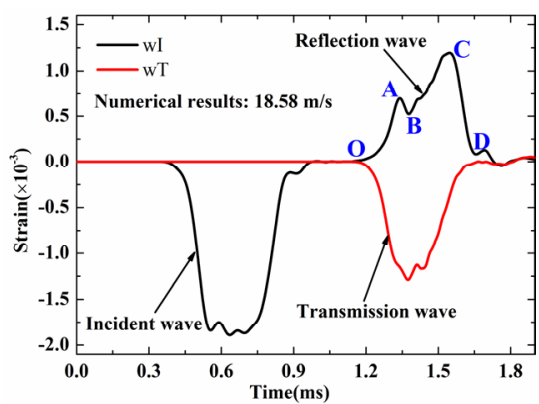

(a) Numerical waveform curves



(b) Experimental waveform curves

Fig. 5. The numerical and experimental waveform curves under the striker velocity of $18.58 \mathrm{~m} / \mathrm{s}$.

\subsection{Comparative analysis of the waveform curves under various strain rates}

The experimental waveform curves under different strain rates are shown in Figure 6 (a), correspondingly, Figure 5 (b) presents the numerical waveform curves which even including the cases of much higher strain rates. In general, the variation tendency of the waveform curves of $\mathrm{C} 1 \sim \mathrm{C} 4$ in the numerical simulation has well reflected the corresponding experimental results with the increasing strain rate. It can further verify the reliability of the waveform curves of $\mathrm{C} 5 \sim \mathrm{C} 7$ in the numerical results.

As shown in Figure 6 (b), the numerical waveform curves of $\mathrm{C} 1 \sim \mathrm{C} 7$ clearly reflect the change from generation to disappearance of the "double peak" phenomenon of the reflection curve with the increasing strain rate. Especially, due to the stress equilibrium condition to guarantee the reliability of SHPB test has been violated in the cases of $\mathrm{C} 6$ and $\mathrm{C} 7$ (it will be discussed a little later), the waveform curves of $\mathrm{C} 6$ and C7 in Figure 6 (b) are plotted with dashed lines, and the "double peak" phenomenon has disappeared in the corresponding reflection curves. After the loss of stress equilibrium, although the strain rate of $\mathrm{C} 7$ is higher than that of C6, the waveforms and amplitude of the transmission curves are nearly the same.



(a) Experimental waveform curves

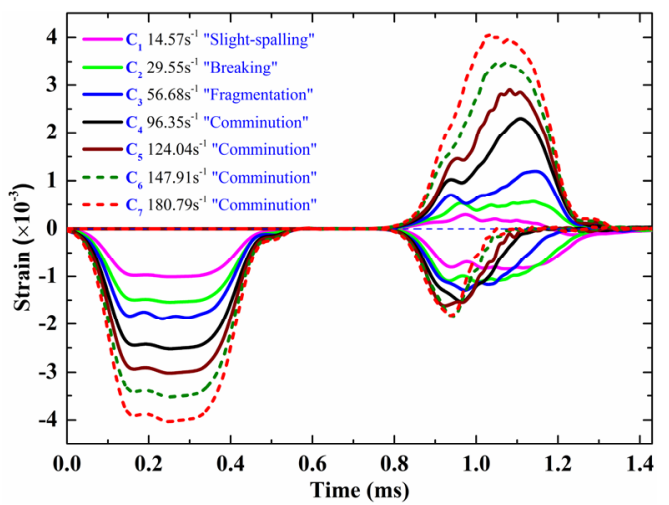

(b) Numerical waveform curves

Fig. 6. Waveform curves under different strain rates.

\subsection{The analysis on the relevant problems of the stress equilibrium}

\subsubsection{The validation of stress equilibrium}

The verification results of the stress equilibrium under various strain rates are shown in Figure 7 (a-f). Apparently, when the loading strain rates are not high, the stress equilibrium can be easily satisfied, such as the cases of the "breaking" and "fragmentation" of the concrete specimen. In the case of "comminution" of the concrete specimen, under the strain rates of $96.35 \mathrm{~s}^{-1}$ and $122.04 \mathrm{~s}^{-1}$ as shown in Figure 7 (c-d), better stress equilibrium is still achieved, and thus the relevant test results are reliable. However, the verification result in Figure 7 (e) shows that the stress equilibrium begins to be 
not satisfied when the loading strain rate up to $147.91 \mathrm{~s}^{-1}$. When the loading strain rate continues up to $180.79 \mathrm{~s}^{-1}$, as shown in Figure 7 (f), the verification result shows that the specimen is really not in stress equilibrium.

Associated with the waveform curves in Figure 6(b), in the SHPB test of the concrete-like material specimen, it can be concluded that the disappearance of the "double peak" phenomenon of the reflection wave can be used as the intuitive criterion of the violation of the stress equilibrium in the specimen in the loading process.

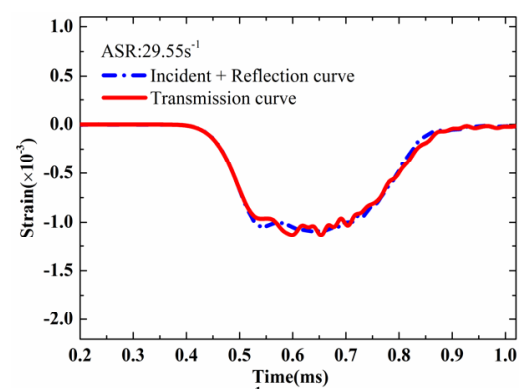

(a) $29.55 \mathrm{~s}^{-1}$, "breaking"

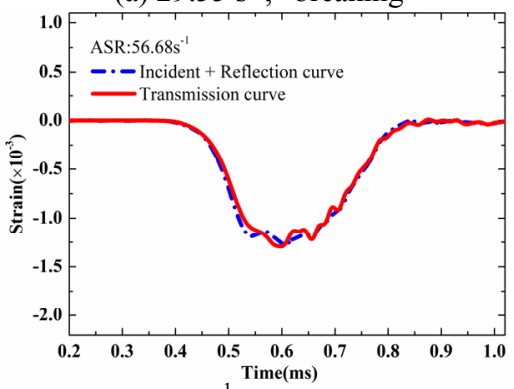

(b) $56.58 \mathrm{~s}^{-1}$, "fragmentation"

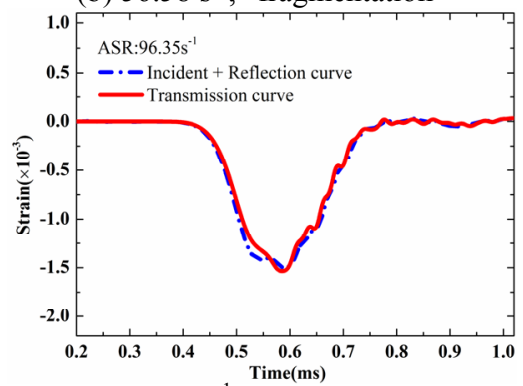

(c) $96.35 \mathrm{~s}^{-1}$, "comminution"

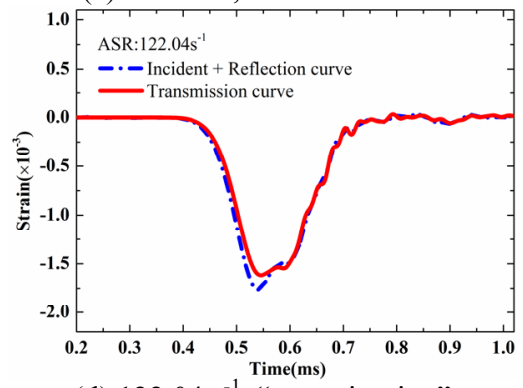

(d) $122.04 \mathrm{~s}^{-1}$, "comminution"

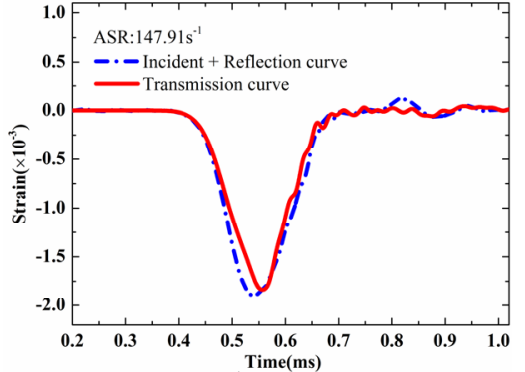

(e) $147.91 \mathrm{~s}^{-1}$, "comminution"

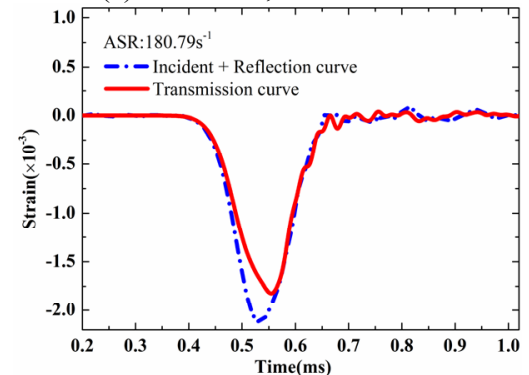

(f) $180.79 \mathrm{~s}^{-1}$, "comminution"

Fig. 7. The verification results of the stress equilibrium under various strain rates.

\subsubsection{The compressive dynamic increase factor (DIF)}

The numerical DIFs of the concrete specimen under different strain rates are given in Figure 8. Obviously, when the stress equilibrium is achieved, the DIF of the concrete specimen increases almost linearly with the increasing strain rate. But, in the case of stress nonequilibrium, the DIF will not increase and maintains at the similar level to that under the critical initiation of the stress non-equilibrium.

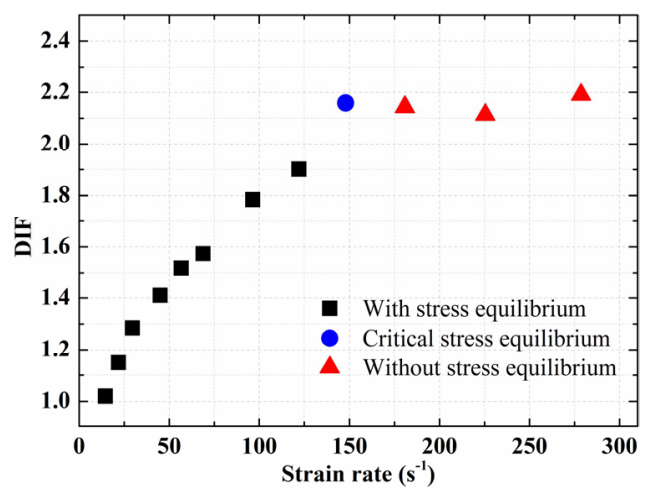

Fig. 8. Numerical DIFs under different strain rates.

Indeed, in the case of stress non-equilibrium, relevant DIFs can't reflect the strain rate effect of the material strength. In this case, the concrete specimen should be regarded as a kind of structure. That is to say, it is the strain rate effect of the material structure strength that the relevant DIFs reflected. Regarding the concrete specimen as a kind of truss structure, its strength should be determined by the material strength. Therefore, the relevant DIFs will not change obviously compared to the value when the stress equilibrium initiates to be violated. 


\subsubsection{The evolution and development of the damage}

In the present numerical SHPB tests, the damage of the concrete specimen under various strain rates is defined as the ratio of the volume of the deleted elements to the total volume of the concrete specimen.

As shown in Figure 9, for the "slight-spalling" of the concrete specimen, the damage develops gradually, and the relevant reflection wave exhibits as a single peak. With the increasing strain rate, the damage from $D_{2}$ to $D_{4}$ develops rapidly, and the relevant reflection waveform curves exhibit as obvious "double peak" phenomenon, as shown in Figure 6 (b). Therefore, it can be concluded that it is the rapid development of the damage of the concrete specimen that leads to the occurrence of the "double peak" phenomenon of the reflection wave under the relatively higher strain rates. This is consistent with the experimental analysis.

Moreover, there is only little difference among $\mathrm{D}_{5}, \mathrm{D}_{6}$ and $D_{7}$. It indicates that the damage extent of the specimen does not increase with the increasing strain rate in the case of stress non-equilibrium, but tends to be a constant value eventually. This is consistent with the discussion on the DIFs.



Fig. 9. The evolution and development processes of the damage of the concrete specimen in the numerical SHPB tests under the different strain rates versus the time.

\section{Conclusions}

For concrete-like damage softening materials, the doublepeak phenomenon in SHPB test can be considered as the specific performance of material. Basically, both experimental analysis and numerical results show that it is caused by the damage of concrete specimen in the loading process. And the "compression wave" phenomenon in the tail of reflection wave exists only in the case that there left large residual specimen after SHPB test. Specially, the disappearance of the "double peak" in the reflection wave can be used as the intuitive indicator of the violation of the stress equilibrium in the SHPB tests for concrete-like damage softening materials. In the case of stress non-equilibrium, the DIF will not increase and maintains at the similar level to that under the critical initiation of the stress non-equilibrium.
The work is supported by the Natural Science Foundations of China (11390361) and the National Outstanding Young Scientist Foundation of China (11225213).

\section{References}

1. Li Q M, Chen X W. Dimensionless formulae for penetration depth of concrete target impacted by a non-deformable projectile [J]. International Journal of Impact Engineering, 28(1):93-116 (2003)

2. Hentz S, Donzé F V, Daudeville L. Discrete element modelling of concrete submitted to dynamic loading at high strain rates [J]. Computers \& Structures, 82(29-30):2509-2524 (2004)

3. Chen $\mathrm{X}, \mathrm{Wu} \mathrm{S}$, Zhou J. Experimental and modeling study of dynamic mechanical properties of cement paste, mortar and concrete $[\mathrm{J}]$. Construction and Building Materials, 47: 419-430 (2013)

4. Zhang M, Wu HJ, Li QM, et al. Further investigation on the dynamic compressive strength enhancement of concrete-like materials based on split Hopkinson pressure bar tests. Part I: Experiments [J]. International Journal of Impact Engineering, 36(12): 1327-1334 (2009)

5. Li QM, Lu YB, Meng H. Further investigation on the dynamic compressive strength enhancement of concrete-like materials based on split Hopkinson pressure bar tests. Part II: numerical simulations [J]. International journal of impact engineering, 36(12): 1335-1345 (2009)

6. Al-Salloum Y, Almusallam T, Ibrahim S M, et al. Rate dependent behavior and modeling of concrete based on SHPB experiments [J]. Cement and Concrete Composites, 55: 34-44 (2015)

7. Hao $Y$, Hao $H$, Jiang $G$ P, et al. Experimental confirmation of some factors influencing dynamic concrete compressive strengths in high-speed impact tests [J]. Cement \& Concrete Research, 52(10):63-70 (2013)

8. Zhou X Q, Hao H. Modelling of compressive behavior of concrete-like materials at high strain rate [J]. International Journal of Solids \& Structures, 45(17):4648-4661 (2008)

9. T.J.Holmquist, G.R. Johnson, W.H.Cook. A computational constitutive model for concrete subjected to large strains, high strain rates, and high pressures. In: The 14th International Symposium on Ballistics, Quebec, pp, 591-600 (1993)

10. Lv T H, Chen X W, Chen G. The 3D Meso-scale Model and Numerical Tests of Split Hopkinson Pressure Bar of Concrete Specimen. Construction and Building Materials, under review (2017)

11. Hao Y F, Zhang X H, Hao H. Numerical analysis of concrete material properties at high strain rate under direct tension [J]. Procedia Engineering, 39(1):51-62 (2012)

12. Hao Y, Hao H, Li Z X. Numerical Analysis of Lateral Inertial Confinement Effects on Impact Test of Concrete Compressive Material Properties. International Journal of Protective Structures, 1(1):145-168 (2010) 